International Journal of Pure and Applied Mathematics

Volume 94 No. 3 2014, 355-372

ISSN: 1311-8080 (printed version); ISSN: 1314-3395 (on-line version)

url: http://www.ijpam.eu

doi: http://dx.doi.org/10.12732/ijpam.v94i3.5

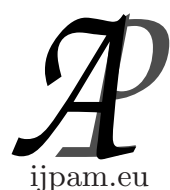

\title{
EXACT SOLUTIONS AND CANONICAL REDUCTION OF THE SELF-DUAL YANG MILLS EQUATIONS TO SOME NONLINEAR EVOLUTION EQUATIONS
}

\author{
A.R. Shehata ${ }^{1 \S}$, J.F.Alzaidy ${ }^{2}$ \\ ${ }^{1}$ Mathematics Department \\ Faculty of Science \\ Minia University, El-Minia, EGYPT \\ ${ }^{2}$ Mathematics Department \\ Faculty of Science \\ Taif University \\ KINGDOM OF SAUDI ARABIA
}

\begin{abstract}
The (constrained) canonical reduction of four-dimensional selfdual Yang-Mills (SDYM) theory to two-dimensional Burgers equation, HunterSaxton equation and Nonlinear diffusion equation are considered. On the other hand, other methods and transformations are developed to obtain exact solutions for the original two dimensional Burgers equation, Hunter-Saxton equation and Nonlinear diffusion. The corresponding gauge potential $A_{\mu}$ and the gauge field strengths $F_{\mu \nu}$ are also obtained.

For these nonlinear evolution equations (NLEEs) which describe pseudospherical surfaces (pss) two new exact solution classes are generated from known solutions by using the Bäcklund transformations with the aid of Mathematica , either the seed solution is constant or a traveling wave.
\end{abstract}

AMS Subject Classification: 35, 53C, 58J, 58Z05

Key Words: SDYM, Burgers equation, Hunter-Saxton equation, nonlinear diffusion equation, Bäcklund transformations

Received: January 2, 2014

(C) 2014 Academic Publications, Ltd. url: www.acadpubl.eu

${ }^{\S}$ Correspondence author 


\section{Introduction}

The self-dual Yang-Mills (SDYM) equations (a system of equations for Lie algebra-valued functions of $C^{4}$ ) play a central role in the field of integrable systems and also play a fundamental role in several other areas of mathematics and physics [1,2]. It arises in relativity [3,4] and in field theory [5]. The SDYM equations describe a connection for a bundle over the Grassmannian of two-dimensional subspaces of the twistor space. Integrability for a SDYM connection means that its curvature vanishes on certain two-planes in the tangent space of the Grassmannian. As shown in [6,7]. This allows one to characterize SDYM connections in terms of the splitting problem for a transition function in a holomorphic bundle over the Riemann sphere, i.e. the trivialization of the bundle $[8,9]$.

The theory of integrable systems has been an active area of mathematics for the past thirty years. Different aspects of the subject have fundamental relations with mechanics and dynamics, applied mathematics, algebraic structures, theoretical physics, analysis including spectral theory and geometry. In recent decades, a class of transformations having their origin in the work by Bäcklund in the late nineteenth century has provided a basis for remarkable advances in the study of nonlinear partial differential equations (NLPDEs)[1-11]. The importance of Bäcklund transformations (BTs) and their generalizations is basically twofold. Thus, on one hand, invariance under a BT may be used to generate an infinite sequence of solutions for certain NLPDEs by purely algebraic superposition principles. On the other hand, BTs may also be used to link certain NLPDEs [12-21] (particularly NLEEs modelling nonlinear waves) to canonical forms whose properties are well known [22-28].

Non-Abelian gauge theories first appeared in the seminal work of Yang and Mills [21] as a non-Abelian generalization of Maxwells equations. Let G be a Lie group (referred to as the gauge group) with Lie algebra (LG) and let $\left\{x_{\mu}\right\}_{\mu=1,2,3,4}$ be coordinates on a four- dimensional manifold $M$ which can be $R^{4}, R^{1,3}$ or $R^{2,2}$. Given the gauge potential $A_{\mu}(x) \in L G$, we introduce the covariant derivatives

$$
D_{\mu}=\partial_{\mu}-A_{\mu},
$$

and their commutators

$$
F_{\mu \nu}=-\left[D_{\mu}, D_{\nu}\right]=\partial_{\mu} A_{\nu}-\partial_{\nu} A_{\mu}-\left[A_{\mu}, A_{\nu}\right],
$$

where $F_{\mu \nu}$ are the gauge field strengths. 
The Yang- Mills equations are a set of coupled, second-order NLPDEs in four dimensions for the LG-valued gauge potential functions $A_{\mu}$ 's, and are extremely difficult to solve in general. It is however possible to obtain a special class of first-order reductions of the full Yang-Mills equations by noting that any $F_{\mu \nu}$ that satisfies

$$
\lambda F_{\mu \nu}={ }^{*} F_{\mu \nu}, \quad \lambda=\left\{\begin{array}{lll} 
\pm 1, & \text { on } & R^{4}, R^{2,2} \\
\pm i, & \text { on } & R^{3,1}
\end{array}\right.
$$

All real solutions of the equations ${ }^{*} F_{\mu \nu}= \pm i F_{\mu \nu}$ are trivial. On $R^{4}$ and $R^{2,2}$, the equations ${ }^{*} F_{\mu \nu}=(-) F_{\mu \nu}$ are called the (anti) SDYM equations. Now consider four complex variables $y, \bar{y}, z$ and $\bar{z}$ defined in [21]

$$
\sqrt{2} y=x_{1}+i x_{2}, \sqrt{2} \bar{y}=x_{1}-i x_{2}, \sqrt{2} z=x_{3}-i x_{4}, \sqrt{2} \bar{z}=x_{3}+i x_{4},
$$

it is simple to check that the self-duality equations $F_{\mu \nu}={ }^{*} F_{\mu \nu}$ reduces to

$$
F_{y z}=0, \quad F_{\bar{y} \bar{z}}=0, \quad F_{y \bar{y}}+F_{z \bar{z}}=0 .
$$

Equations (5) are the compatibility condition of the linear problem [21]

$$
\begin{aligned}
& \left(\psi_{y}+i \zeta \psi_{\bar{z}}\right)=\left(A_{y}+i \zeta A_{\bar{z}}\right) \psi, \\
& \left(\psi_{z}-i \zeta \psi_{\bar{y}}\right)=\left(A_{y}-i \zeta A_{\bar{y}}\right) \psi,
\end{aligned}
$$

where $\zeta$ is a parameter, independent of $y, \bar{y}, z$ and $\bar{z}$.

The compatibility condition is simply

$$
\left(\partial_{z}-i \zeta \partial_{\bar{y}}\right)\left(\partial_{y}+i \zeta \partial_{\bar{z}}\right) \psi=\left(\partial_{y}+i \zeta \partial_{\bar{z}}\right)\left(\partial_{z}-i \zeta \partial_{\bar{y}}\right) \psi
$$

On using equations (6) and (7), this gives

$$
\left[F_{y z}-i \zeta\left(F_{y \bar{y}}+F_{z \bar{z}}\right)-\zeta^{2} F_{\bar{y} \bar{z}}\right] \psi=0 .
$$

Equations (5) can be immediately integrated, since they are pure gauge, to give

$$
A_{y}=D^{-1} D_{y}, A_{z}=D^{-1} D_{z}, A_{\bar{y}}=\bar{D}^{-1} \bar{D}_{\bar{y}}, A_{\bar{z}}=\bar{D}^{-1} \bar{D}_{\bar{z}}
$$

where $D$ and $\bar{D}$ are arbitrary $2 \times 2$ complex matrix functions of $y, \bar{y}, z$ and $\bar{z}$ , and with determinant $=1$ (for $\mathrm{SU}(2)$ gauge group) and $D_{y}=\partial_{y} D$, etc. For 
real gauge fields $A_{\mu} \doteq-A_{\mu}^{+}$(the symbol $\doteq$ is used for equations valid only for real values of $x_{1}, x_{2}, x_{3}$ and $\left.x_{4}\right)$, we require

$$
\bar{D} \doteq\left(D^{+}\right)^{-1} .
$$

Gauge transformations are the transformations

$$
D \rightarrow D U, \bar{D} \rightarrow \bar{D} U, U^{+} U \doteq I
$$

where $U$ is a $2 \times 2$ matrix function of $y, \bar{y}, z, \bar{z}$ with determined $=1$. Under transformation (12), equation (11) remains unchanged. We now define the hermitian matrix as

$$
\jmath \equiv D \bar{D}^{-1} \doteq D D^{+}
$$

$\jmath$ has the very important property of being invariant under the gauge transformation (12). The only non vanishing field strengths in terms of $\jmath$ becomes

$$
F_{u \bar{v}}=-\bar{D}^{-1}\left(\jmath^{-1} \jmath_{u}\right)_{\bar{v}} \bar{D},
$$

$(u, v=y, z)$ and the remaining self-duality equation (5) takes the form

$$
\left(\jmath^{-1} \jmath_{y}\right)_{\bar{y}}+\left(\jmath^{-1} \jmath_{z}\right)_{\bar{z}}=0 .
$$

The action density in terms of $\jmath$ is

$$
\begin{aligned}
\phi(\jmath) & =-\frac{1}{2} \operatorname{Tr} F_{\mu \nu} F_{\mu \nu} \\
& =-2 \operatorname{Tr}\left(F_{y \bar{y}} F_{z \bar{z}}+F_{y \bar{z}} F_{\bar{y} z}\right) \\
& =-2 \operatorname{Tr}\left(\left(\jmath^{-1} \jmath_{y}\right)_{\bar{y}}\left(J^{-1} \jmath_{z}\right)_{\bar{z}}-\left(J^{-1} \jmath_{y}\right)_{\bar{z}}\left(J^{-1} \jmath_{z}\right)_{\bar{y}}\right)
\end{aligned}
$$

In this paper, the canonical reduction of four dimensional self-dual Yang-Mills theory to two dimensional Burgers equation [15], Hunter -Saxton (HS) equation [16] and Nonlinear diffusion equation [17] are considered. We give a new of exact solution for the Burgers equation ,HS equation and Nonlinear diffusion equation by applying the BTs method with the aid of Mathematica [18-29]. Consequently we find exact solutions for self-dual Yang Mills equations. In addition the corresponding gauge potential $A_{\mu}$ and the gauge field strengths $F_{\mu \nu}$ are also obtained.

The paper is organized as follows: On one hand the reduction of Yang-Mills theory to Burgers equation, HS equation, Nonlinear diffusion equation and exact solutions are presented in Sections 2, 3 and 4 respectively. Moreover the gauge potential $A_{\mu}$ and the gauge field strengths $F_{\mu \nu}$ are also obtained. Sections 5 contains the conclusion. 


\section{The Canonical Reduction of Four-Dimensional SDYM Theory to} Two-Dimensional Burgers Equation, HS Equation and Nonlinear Diffusion Equation

Suppose that $A_{\mu}$ 's depend on $x=\bar{y}$ and $t=\bar{z}$ only. If we use a gauge in which $A_{y}=0$, in terms of the matrix-valued functions $P:=A_{z}, Q:=A_{\bar{y}}, R:=A_{\bar{z}}$, the SDYM equations (5) are

$$
\begin{gathered}
P_{t}+[P, R]=0, \\
R_{x}-Q_{t}-[Q, R]=0 .
\end{gathered}
$$

Examples of Reductions

\section{Burgers equation}

Let $p$ take the canonical form

$$
P=\left(\begin{array}{cc}
0 & k \\
-k & 0
\end{array}\right),
$$

for some constant $k$. We then find that

$$
\begin{gathered}
R=\left(\begin{array}{cc}
0 & u_{x}+\frac{1}{2} u^{2}+h(x) \\
-\left(u_{x}+\frac{1}{2} u^{2}+h(x)\right) & 0
\end{array}\right), \\
Q=\left(\begin{array}{cc}
0 & u \\
-u & 0
\end{array}\right)
\end{gathered}
$$

from Eq. (18), we obtain the Burgers equation

$$
u_{t}=u_{x x}+u u_{x}+h_{x}(x)
$$

\section{HS equation}

Let $p$ take the canonical form

$$
P=\left(\begin{array}{cc}
0 & k \\
-k & 0
\end{array}\right),
$$

for some constant $k$. We then find that

$$
\begin{gathered}
R=\left(\begin{array}{cc}
0 & -\left(u_{x x} u+\frac{1}{2} u_{x}^{2}\right) \\
u_{x x} u+\frac{1}{2} u_{x}^{2} & 0
\end{array}\right), \\
Q=\left(\begin{array}{cc}
0 & u_{x x} \\
-u_{x x} & 0
\end{array}\right),
\end{gathered}
$$


from Eq. (18), we obtain the Burgers equation

$$
u_{x x t}=-u_{x x x} u-2 u_{x x} u_{x}
$$

\section{Nonlinear diffusion equation}

Let $p$ take the canonical form

$$
P=\left(\begin{array}{cc}
0 & 0 \\
k & 0
\end{array}\right)
$$

for some constant $k$. We then find that

$$
\begin{gathered}
R=\left(\begin{array}{cc}
0 & 0 \\
\frac{u_{x}}{u^{2}}-x & 0
\end{array}\right), \\
Q=\left(\begin{array}{ll}
0 & 0 \\
u & 0
\end{array}\right),
\end{gathered}
$$

from Eq. (18), we obtain the Burgers equation

$$
u_{t}=u_{x x} u^{-2}-2 u_{x}^{2} u^{-3}-1 .
$$

\section{The AKNS System for Some NLEEs which Describe PSS and its BTs}

We recall the definition $[16,23]$ of a differential equation (DE) that describes a pss. $M^{2}$ Let be a two dimensional differentiable manifold with coordinates $(x, t)$. A DE for a real function $u(x, t)$ describes a pss if it is a necessary and sufficient condition for the existence of differentiable functions

$$
f_{i j}, \quad 1 \leq i \leq 3, \quad 1 \leq j \leq 2,
$$

depending on $u$ and its derivatives such that the one-forms

$$
\omega_{1}=f_{11} d x+f_{12} d t, \omega_{2}=f_{21} d x+f_{22} d t, \omega_{3}=f_{31} d x+f_{32} d t,
$$

satisfy the structure equations of a pss, i.e.,

$$
d \omega_{1}=\omega_{3} \wedge \omega_{2}, \quad d \omega_{2}=\omega_{1} \wedge \omega_{3}, \quad d \omega_{3}=\omega_{1} \wedge \omega_{2} .
$$

As a consequence, each solution of the DE provides a local metric on $M^{2}$, whose Gaussian curvature is constant, equal to -1 . Moreover, the above definition is 
equivalent to saying that $\mathrm{DE}$ for $u$ is the integrability condition for the problem $[14,27]$ :

$$
d \phi=\Omega \phi, \quad \phi=\left(\begin{array}{c}
\phi_{1} \\
\phi_{2}
\end{array}\right),
$$

where $d$ denotes exterior differentiation, $\phi$ is a column vector and the $2 \times 2$ matrix $\Omega\left(\Omega_{i j} i, j=1,2\right)$ is traceless

$$
\Omega=\frac{1}{2}\left(\begin{array}{cc}
\omega_{2} & \omega_{1}-\omega_{3} \\
\omega_{1}+\omega_{3} & -\omega_{2}
\end{array}\right),
$$

Take

$$
\Omega=\left(\begin{array}{cc}
\frac{\eta}{2} d x+A d t & q d x+B d t \\
r d x+C d t & -\frac{\eta}{2} d x-A d t
\end{array}\right)=S d x+T d t,
$$

from Eqs. (34) and (35), we obtain

$$
\phi_{x}=S \phi, \quad \phi_{t}=T \phi,
$$

where $S$ and $T$ are two $2 \times 2$ null-trace matrices

$$
\begin{aligned}
& S=\left(\begin{array}{cc}
\frac{\eta}{2} & q \\
r & -\frac{\eta}{2}
\end{array}\right), \\
& T=\left(\begin{array}{cc}
A & B \\
C & -A
\end{array}\right) .
\end{aligned}
$$

Here $\eta$ is a parameter, independent of $x$ and $t$, while $q$ and $r$ are functions of $x$ and $t$. Now

$$
0=d^{2} \phi=d \Omega \phi-\Omega \wedge d \phi=(d \Omega-\Omega \wedge \Omega) \phi
$$

which requires the vanishing of the two form

$$
\Theta \equiv d \Omega-\Omega \wedge \Omega=0,
$$

or in component form

$$
\begin{array}{r}
-A_{x}+q C-r B=0, \\
q_{t}-2 A q-B_{x}+\eta B=0 \\
r_{t}-C_{x}+2 A r-\eta C=0
\end{array}
$$

Chern and Tenenblat [10] obtained Eq. (40) directly from the structure equations (33). By suitably choosing $r, A, B$ and $C$ in (40), we shall obtain 
various NLEEs which $q$ must satisfy. Konno and Wadati introduced the function $[30]$

$$
\Gamma=\frac{\phi_{1}}{\phi_{2}}
$$

this function first appeared used and explained in the geometric context of pss equations in [11,13], and see also the classical papers by Sasaki [31] and Chern-Tenenblat [10]. Then Eq. (36) is reduced to the Riccati equations:

$$
\begin{aligned}
& \frac{\partial \Gamma}{\partial x}=\eta \Gamma-r \Gamma^{2}+q \\
& \frac{\partial \Gamma}{\partial t}=2 A \Gamma-C \Gamma^{2}+B
\end{aligned}
$$

Our procedure in the following is that we construct a transformation $\Gamma^{\prime}$ satisfying the same equation as (42) and (43) with a potential $u^{\prime}$ where

$$
u^{\prime}=u+f(\Gamma, \eta)
$$

Chern and Tenenblat [10] introduced several examples of (44) for pss equations. For use in the sequel, we list the Burgers equation ; HS equation; Nonlinear diffusion equation and their corresponding $\mathrm{BT}$ in the following.

\subsection{Burgers Equation}

We have

$$
\begin{aligned}
& \omega_{1}=\left(\frac{1}{2} u-\frac{\beta}{\eta}\right) d x+\left(\frac{1}{2} u_{x}+\frac{1}{4} u^{2}+\frac{1}{2} h(x)\right) d t \\
& \omega_{2}=\eta d x+\left(\frac{\eta}{2} u+\beta\right) d t \\
& \omega_{3}=-\eta d x-\left(\frac{\eta}{2} u+\beta\right) d t
\end{aligned}
$$

in which $\eta \neq 0$ is a parameter, and $\beta$ is a solution of the equation

$$
\beta^{2}-\eta \beta_{x}+\left(\frac{\eta^{2}}{2}\right) h(x)
$$

For any solution $u(x, t)$ of the Burgers equation (22), the matrices $S$ and $T$ are 


$$
\begin{gathered}
S=\left(\begin{array}{cc}
\frac{\eta}{2} & \frac{1}{2}\left(\frac{u}{2}-\frac{\beta}{\eta}+\eta\right) \\
\frac{1}{2}\left(\frac{u}{2}-\frac{\beta}{\eta}-\eta\right) & -\frac{\eta}{2}
\end{array}\right), \\
T=\left(\begin{array}{cc}
\frac{1}{2}\left(\frac{\eta}{2} u+\beta\right) & \frac{1}{2}\left(\frac{u x}{2}+\frac{u^{2}}{4}+\frac{h(x)}{2}+\frac{\eta}{2} u+\beta\right) \\
\frac{1}{2}\left(\frac{u x}{2}+\frac{u^{2}}{4}+\frac{h(x)}{2}-\frac{\eta}{2} u-\beta\right) & -\frac{1}{2}\left(\frac{\eta}{2} u+\beta\right)
\end{array}\right),
\end{gathered}
$$

the above matrices $S, T$ satisfy Eqs. (40). Then Eq. (42) becomes

$$
\frac{\partial \Gamma}{\partial x}=\eta \Gamma+\frac{1}{2}\left(\frac{u}{2}-\frac{\beta}{\eta}+\eta\right)-\frac{1}{2}\left(\frac{u}{2}-\frac{\beta}{\eta}-\eta\right) \Gamma^{2} .
$$

If we choose $\Gamma^{\prime}$ and $u^{\prime}$ as [23]

$$
\begin{gathered}
\Gamma^{\prime}=\frac{1}{\Gamma}, \\
u^{\prime}=-u+4 \frac{\beta}{\Gamma}+8 \frac{\partial}{\partial x} \tanh ^{-1} \Gamma .
\end{gathered}
$$

\subsection{HS Equation}

We have

$$
\begin{aligned}
& \omega_{1}=\left(u_{x x}-\beta\right) d x+\left(\frac{u_{x}-u_{x} \beta}{\eta}+\frac{1-\beta}{\eta^{2}}-u u_{x x}-1+u \beta\right) d t, \\
& \omega_{2}=\eta d x+\left(\frac{1-\beta}{\eta}-\eta u+u_{x}\right) d t, \\
& \omega_{3}=\left(u_{x x}+1\right) d x+\left(\frac{u_{x}-u_{x} \beta}{\eta}+\frac{1-\beta}{\eta^{2}}-u u_{x x}-u\right) d t,
\end{aligned}
$$

in which the parameter $\eta$ and $\beta$ are constrained by the relation $\eta^{2}+\beta^{2}=1$. For any solution $u(x, t)$ of the HS equation (26), the matrices $S$ and $T$ are

$$
S=\left(\begin{array}{cc}
\frac{\eta}{2} & -\frac{1}{2}(\beta+1) \\
\frac{1}{2}\left(2 u_{x x}-\beta+1\right) & -\frac{\eta}{2}
\end{array}\right),
$$




$$
T=\left(\begin{array}{cc}
\frac{1}{2}\left(\frac{1-\beta}{\eta}-\eta u+u_{x}\right) & \frac{1}{2}(u \beta+u-1) \\
\frac{1}{2}\left(\frac{u_{x}-u_{x} \beta}{\eta}+\frac{1-\beta}{\eta^{2}}-u u_{x x}-\frac{u \beta}{2}-\frac{1}{2} u-\frac{1}{2}\right) & -\frac{1}{2}\left(\frac{1-\beta}{\eta}-\eta u+u_{x}\right)
\end{array}\right),
$$

the above matrices $S, T$ satisfy Eqs. (40). Then Eq. (42) becomes

$$
\frac{\partial \Gamma}{\partial x}=\eta \Gamma-\frac{1}{2}(\beta+1)-\frac{1}{2}\left(2 u_{x x}-\beta+1\right) \Gamma^{2} .
$$

If we choose $\Gamma^{\prime}$ and $u^{\prime}$ as [23]

$$
\Gamma^{\prime}=-\Gamma
$$

we have

$$
v^{\prime}=v-\frac{2}{\Gamma}, \quad v^{\prime}=u_{x}^{\prime}, \quad v=u_{x}, \quad \Gamma \neq 0
$$

\subsection{Nonlinear Diffusion Equation}

We have

$$
\begin{aligned}
& \omega_{1}=-e^{-\epsilon \eta x} u d x+\left(-e^{-\epsilon \eta x} \frac{u_{x}}{u^{2}}+\delta(x)\right) d t \\
& \omega_{2}=\eta d x \\
& \omega_{3}=\epsilon \omega_{1}
\end{aligned}
$$

in which $\epsilon= \pm 1$ and $\delta(x)$ is a solution of the equation $\epsilon \eta \delta+\delta_{x}=e^{-\epsilon \eta x}$.

For any solution $u(x, t)$ of the Nonlinear diffusion equation (30), the matrices $S$ and $T$ are

$$
\begin{gathered}
S=\left(\begin{array}{cc}
\frac{\eta}{2} & \frac{\epsilon-1}{2} e^{-\epsilon \eta x} u \\
-\frac{\epsilon+1}{2} e^{-\epsilon \eta x} u & -\frac{\eta}{2}
\end{array}\right), \\
0 \\
0=\left(\begin{array}{cc}
\frac{\epsilon-1}{2}\left(e^{-\epsilon \eta x} \frac{u_{x}}{u^{2}}-\delta(x)\right. \\
0
\end{array}\right),
\end{gathered}
$$

the above matrices $S, T$ satisfy Eqs. (40). Then Eq. (42) becomes

$$
\frac{\partial \Gamma}{\partial x}=\eta \Gamma+\frac{\epsilon-1}{2} e^{-\epsilon \eta x} u+\frac{\epsilon+1}{2} e^{-\epsilon \eta x} u \Gamma^{2} .
$$


If we choose $\Gamma^{\prime}$ and $u^{\prime}$ as $[23]$

$$
\Gamma^{\prime}=\Gamma
$$

we have

$$
u^{\prime}=-u+\frac{4 e^{-\epsilon \eta x}\left(\Gamma_{x}-\eta \Gamma\right)}{\epsilon\left(\Gamma^{2}+1\right)+\left(\Gamma^{2}-1\right)}, \quad \Gamma \neq \pm 1 .
$$

\section{The Known Solution is a Constant $u_{0}$}

\subsection{Burgers Equation}

Substitute $u=u_{0}$ into the matrices $S$ and $T$ in (46) and (47), then by (36) we have

$$
d \phi=\phi_{x} d x+\phi_{t} d t=S \phi d \rho
$$

where

$$
\begin{gathered}
S=\left(\begin{array}{cc}
\frac{\eta}{2} & \frac{1}{2}\left(\frac{u_{0}}{2}-\frac{\beta}{\eta}+\eta\right) \\
\frac{1}{2}\left(\frac{u_{0}}{2}-\frac{\beta}{\eta}-\eta\right) & -\frac{\eta}{2}
\end{array}\right), \\
\rho=x+b t, \quad b=\frac{u_{0}}{2}+\frac{\beta}{\eta} .
\end{gathered}
$$

The solution of Eq. (60) is

$$
\phi=e^{s \rho} \phi_{0}=\left(1+\rho s+\frac{\rho^{2} s^{2}}{2 !}+\frac{\rho^{3} s^{3}}{3 !}+\ldots\right) \phi_{0},
$$

where $\phi_{0}$ is a constant column vector. The solution of Eq. (63) is

$$
\begin{aligned}
\phi & =\left(\begin{array}{cc}
\cosh (\alpha \rho)+\frac{\eta}{2 \alpha} \sinh (\alpha \rho) & \left(1+\frac{\eta}{2 \alpha}\right) \sinh (\alpha \rho) \\
\left(1-\frac{\eta}{2 \alpha}\right) \sinh (\alpha \rho) & \cosh (\alpha \rho)-\frac{\eta}{2 \alpha} \sinh (\alpha \rho)
\end{array}\right)^{\phi_{0},} \\
\alpha & =\frac{-2 \beta+\eta u_{0}}{4 \eta}
\end{aligned}
$$

Now, we choose $\phi_{0}=(1,0)^{T}$ in $(64)$, then we have

$$
\phi=\left(\begin{array}{c}
\cosh (\alpha \rho)+\frac{\eta}{2 \alpha} \sinh (\alpha \rho) \\
\left(1-\frac{\eta}{2 \alpha}\right) \sinh (\alpha \rho)
\end{array}\right) .
$$


Substitute (65) into (41), then by (49), we obtain the new solutions of the Burgers equation (22)

$$
u^{\prime}=-u_{0}+\frac{4 \beta}{\eta}+8 \frac{\partial}{\partial x} \tanh ^{-1}\left(\frac{\eta+2 \alpha \cosh (\alpha \rho)}{2 \alpha-\eta}\right) .
$$

We can calculate the gauge potential $A_{\mu}$ and the gauge field strengths $F_{\mu \nu}$ from equations (6)-(10) and (19)-(21), then

$$
\begin{array}{lc}
A_{y}=0, \quad A_{\bar{y}}=\left(\begin{array}{cc}
0 & u^{\prime} \\
-u^{\prime} & 0
\end{array}\right), & A_{z}=\left(\begin{array}{cc}
0 & k \\
-k & 0
\end{array}\right), \\
A_{\bar{z}}=\left(\begin{array}{cc}
0 & u_{x}^{\prime}+\frac{1}{2} u^{\prime 2}+h(x) \\
-\left(u_{x}^{\prime}+\frac{1}{2} u^{\prime 2}+h(x)\right) & 0
\end{array}\right)
\end{array}
$$

Consequently, we obtain the gauge field strengths $F_{\mu \nu}$ as follows:

$$
\begin{gathered}
F_{y z}=-\left[A_{y}, A_{z}\right], \quad F_{\bar{y} \bar{z}}=\partial_{x} A_{\bar{z}}-\partial_{t} A_{\bar{y}}-\left[A_{\bar{y}}, A_{\bar{z}}\right], \\
F_{y \bar{y}}=-\partial_{x} A_{y}-\left[A_{y}, A_{\bar{y}}\right], \quad F_{z \bar{z}}=\partial_{t} A_{z}-\left[A_{z}, A_{\bar{z}}\right]
\end{gathered}
$$

\subsection{HS Equation}

Substitute $u=u_{0}$ into the matrices $S$ and $T$ in (51) and (52), then by (36) we have

$$
d \phi=\phi_{x} d x+\phi_{t} d t=S \phi d \rho
$$

where

$$
\begin{gathered}
S=\left(\begin{array}{cc}
\frac{\eta}{2} & -\frac{1}{2}(\beta+1) \\
\frac{1}{2}(-\beta+1) & -\frac{\eta}{2}
\end{array}\right)^{\prime} \\
\rho=x+m t, \quad m=\frac{1}{1+\beta}-u_{0} .
\end{gathered}
$$

The solution of Eq. (69) is

$$
\phi=e^{s \rho} \phi_{0}=\left(1+\rho s+\frac{\rho^{2} s^{2}}{2 !}+\frac{\rho^{3} s^{3}}{3 !}+\ldots\right) \phi_{0},
$$

where $\phi_{0}$ is a constant column vector. The solution of Eq. (72) is

$$
\phi=\left(\begin{array}{cc}
1+\frac{1}{2} \rho \eta & \frac{-(1+\beta)}{2} \rho \\
\frac{1-\beta}{2} \rho & 1-\frac{1}{2} \rho \eta
\end{array}\right)^{\phi_{0} .}
$$


Now, we choose $\phi_{0}=(1,0)^{T}$ in $(57)$, then we have

$$
\phi=\left(\begin{array}{c}
1+\frac{1}{2} \rho \eta \\
\frac{1-\beta}{2} \rho
\end{array}\right) .
$$

Substitute (74) into (41), then by (54), we obtain the new solutions of the HS equation (26)

$$
v^{\prime}=v_{0}+\frac{2(-1+\beta) \rho}{2+\eta \rho} .
$$

We can calculate the gauge potential $A_{\mu}$ and the gauge field strengths $F_{\mu \nu}$ from equations (6)-(10) and (23)-(25), then

$$
\begin{aligned}
& A_{y}=0, \quad A_{\bar{y}}=\left(\begin{array}{cc}
0 & u_{x x}^{\prime} \\
-u_{x x}^{\prime} & 0
\end{array}\right), \quad A_{z}=\left(\begin{array}{cc}
0 & k \\
-k & 0
\end{array}\right) \text {, } \\
& A_{\bar{z}}=\left(\begin{array}{cc}
0 & -\left(u_{x x}^{\prime} u^{\prime}+\frac{1}{2} u_{x}^{\prime 2}\right) \\
u_{x x}^{\prime} u^{\prime}+\frac{1}{2} u_{x}^{\prime 2} & 0
\end{array}\right)
\end{aligned}
$$

Consequently, we obtain the gauge field strengths $F_{\mu \nu}$ as follows:

$$
\begin{gathered}
F_{y z}=-\left[A_{y}, A_{z}\right], \quad F_{\bar{y} \bar{z}}=\partial_{x} A_{\bar{z}}-\partial_{t} A_{\bar{y}}-\left[A_{\bar{y}}, A_{\bar{z}}\right], \\
F_{y \bar{y}}=-\partial_{x} A_{y}-\left[A_{y}, A_{\bar{y}}\right], \quad F_{z \bar{z}}=\partial_{t} A_{z}-\left[A_{z}, A_{\bar{z}}\right]
\end{gathered}
$$

\subsection{Nonlinear Diffusion Equation}

Substitute $u=u_{0}, u_{0} \neq 0$ into the matrices $S$ and $T$ in (56) and (57), then by (36) we have

$$
d \phi=\phi_{x} d x+\phi_{t} d t=S \phi d \rho
$$

where

$$
\begin{gathered}
S=\left(\begin{array}{cc}
\frac{\eta}{2} & \frac{\epsilon-1}{2} e^{-\epsilon \eta x} u_{0} \\
-\frac{\epsilon+1}{2} e^{-\epsilon \eta x} u_{0} & -\frac{\eta}{2}
\end{array}\right), \\
\rho=x+\gamma t, \quad \gamma=\frac{-\delta(x)}{u_{0}} e^{-\epsilon \eta x}, \quad \lambda \delta(x)=0
\end{gathered}
$$

The solution of Eq. (62) is

$$
\phi=e^{s \rho} \phi_{0}=\left(1+\rho s+\frac{\rho^{2} s^{2}}{2 !}+\frac{\rho^{3} s^{3}}{3 !}+\ldots\right) \phi_{0},
$$


where $\phi_{0}$ is a constant column vector. The solution of Eq. (64) is

$$
\phi=\left(\begin{array}{cc}
\cosh \left(\frac{\eta}{2} \rho\right)+\sinh \left(\frac{\eta}{2} \rho\right) & \frac{(\epsilon-1) u_{0}}{\eta} e^{-\epsilon \eta x} \sinh \left(\frac{\eta}{2} \rho\right) \\
\frac{-(\epsilon+1) u_{0}}{\eta} e^{-\epsilon \eta x} \sinh \left(\frac{\eta}{2} \rho\right) & \cosh \left(\frac{\eta}{2} \rho\right)+\sinh \left(\frac{\eta}{2} \rho\right)
\end{array}\right) \phi_{0} .
$$

Now, we choose $\phi_{0}=(1,0)^{T}$ in $(81)$, then we have

$$
\phi=\left(\begin{array}{c}
\cosh \left(\frac{\eta}{2} \rho\right)+\sinh \left(\frac{\eta}{2} \rho\right) \\
\frac{-(\epsilon+1) u_{0}}{\eta} e^{-\epsilon \eta x} \sinh \left(\frac{\eta}{2} \rho\right)
\end{array}\right) .
$$

Substitute (82) into (41), then by (59), we obtain the new solutions of Nonlinear diffusion equation (30)

$$
u^{\prime}=\left(\frac{\left[\left(1+\cosh \left(\frac{\eta}{2} \rho\right)\right)\left(-3+\cosh \left(\frac{\eta}{2} \rho\right)+4 \epsilon\right)\right]-2 \operatorname{csch}^{2}\left(\frac{\eta}{2} \rho\right)}{\left(1+\cosh \left(\frac{\eta}{2} \rho\right)\right)^{2}}\right) \eta^{2} u_{0} .
$$

We can calculate the gauge potential $A_{\mu}$ and the gauge field strengths $F_{\mu \nu}$ from equations (6)-(10) and (27)-(29), then

$$
A_{y}=0, A_{\bar{y}}=\left(\begin{array}{cc}
0 & 0 \\
-u^{\prime} & 0
\end{array}\right), A_{z}=\left(\begin{array}{ll}
0 & 0 \\
k & 0
\end{array}\right), A_{\bar{z}}=\left(\begin{array}{cc}
0 & 0 \\
\frac{u_{x}^{\prime}}{u^{\prime 2}}-x & 0
\end{array}\right) .
$$

Consequently, we obtain the gauge field strengths $F_{\mu \nu}$ as follows:

$$
\begin{gathered}
F_{y z}=-\left[A_{y}, A_{z}\right], \quad F_{\bar{y} \bar{z}}=\partial_{x} A_{\bar{z}}-\partial_{t} A_{\bar{y}}-\left[A_{\bar{y}}, A_{\bar{z}}\right] \\
F_{y \bar{y}}=-\partial_{x} A_{y}-\left[A_{y}, A_{\bar{y}}\right], \quad F_{z \bar{z}}=\partial_{t} A_{z}-\left[A_{z}, A_{\bar{z}}\right]
\end{gathered}
$$

\section{Conclusions}

In this paper, we considered the construction of exact solutions to Burgers equation, HS equation and Nonlinear diffusion equation. We obtain traveling wave solutions for the above equations by using BTs method with the aid of Mathematica.

The soliton phenomena and integrable NLEEs represent an important and well established field of modern physics, mathematical physics and applied mathematics. Solitons are found in various areas of physics from hydrodynamics and plasma physics, nonlinear optics and solid state physics, to field theory 
and gravitation. NLEEs which describe soliton phenomena have an universal character.

A traveling wave of permanent form has already been met; this is the solitary wave solution of the NLEE itself. Such a wave is a special solution of the governing equation which does not change its shape and which propagates at constant speed. The SDYM equations play a central role in the field of integrable systems and also play a fundamental role in several other areas of mathematics and physics.

In addition the SDYM equations are a rich source of integrable systems suggested by the fact that they are the compatibility condition of an associated linear problem which admits enormous freedom if one allows the associated gauge algebra to be arbitrary. The classical soliton equations in $1+1,2+1$ and $3+1$ dimensions are reductions of the SDYM equations with finite-dimensional gauge algebra. In this paper we have demonstrated the reductions of the SDYM equations to Burgers equation ; HS equation and Nonlinear diffusion equation and also obtained traveling wave solution.

\section{References}

[1] J.P. Bourguignon, H.B. Lawson and J. Simons, Stability and gap Phenomena for Yang-Mills fields, Proc.Nat.Acad Sci., Vol.76,(1979), pp.15501553.

[2] R. Donagi and E. Witten, Supersymmetric Yang-Mills theory and integrable systems, Nucl. Phys., Vol.B460,No.2,(1996), pp.299-334.

[3] W. Klingenberg, Riemannian Geometry,Walter de Gruyter, Berlin, New York, 1982.

[4] T. Chuu-Lian and K. Uhlenbeck, Introduction to Surveys in Differential Geometry: Integrable Systems, J. Differential Geometry, Vol.4, 5(1999).

[5] M. J. Abolwitz and P.A. Clarkson, Solitons, Nonlinear Evolution Equation and Inverse Scattering, (Cambridge University Press, Cambridge,1991).

[6] A.H. Khater , D.K. Callebaut, A.A. Abdalla, A.R. Shehata and S.M. Sayed, Bäcklund transformations and exact solutions for self-dual SU(3) Yang-Mills equations, IL Nuovo Cimento , Vol.B114,No1,(1999), pp.1-10.

[7] A.H. Khater, A.R. Shehata, D.K. Callebaut and S.M. Sayed, Self-Dual Solutions for SU(2) and SU(3) Gauge Fields on Euclidean Space, International J. of Theoretical Physics, Vol.43,(2004), pp.151-159. 
[8] R. Hermann, Vector Bundles in Mathematical Physics, Vol. I and II(W.A. Benjamin, Inc.) (1970).

[9] N. Hitchin , Stable bundles and integrable systems, Duke Math. Journal , Vol.54, (1987),pp.91-114.

[10] S.S. Chern and K. Tenenblat, Pseudospherical surfaces and evolution equations , Stud. Appl. Math., Vol.74, No.1,(1986),pp.55-83.

[11] J.A. Cavalcante and K. Tenenblat, Conservation laws for nonlinear evolution equations, J. Math. Phys., Vol.29, No.4,(1988),pp.1044-1049.

[12] R. Beals, M. Rabelo and K. Tenenblat, Bäcklund transformations and inverse scattering solutions for some pseudo-spherical surfaces, Stud. Appl. Math., Vol.81,(1989), pp.125-151.

[13] E.G. Reyes, Conservation laws and Calapso-Guichard deformations of equations describing pseudo-spherical surfaces, J. Math. Phys., Vol.41,(2000), pp.2968-2988.

[14] E.G. Reyes, On Geometrically integrable equations and Hierarchies of pseudo-spherical type, Contemporary Mathematics, Vol.285,(2001), pp.145-155.

[15] E.G. Reyes, The Soliton Content of the Camassa-Holm and Hunter-Saxton Equations, Proceedings of Institute of Mathematics of NAS of Ukraine, Vol.43,(2002), pp.201-208.

[16] A.H. Khater, D.K. Callebaut and S.M. Sayed, Conservation laws for some nonlinear evolution equations which describe pseudo-spherical surfaces, J. Geometry and Phys., Vol.51,(2004), pp.332-352.

[17] M. V. Foursov, P. J. Olver and E. G. Reyes, On formal integrability of evolution equations and local geometry of surfaces, Differential Geometry and its applications, Vol.15,(2001), pp.183-199.

[18] E. Witten , Two dimensional gravity and intersection theory on moduli space, Survey in Differential Geometry, Vol.1,(1991), pp.243-310.

[19] K. Chadan and P.C. Sabatier, Inverse problem in quantum scattering theory , (springer, New York)( 1977). 
[20] M.J. Ablowitz, D.J. Kaup, A.C. Newell and H. Segur, The inverse scattering transform-Fourier analysis for nonlinear problems, Stud. Appl. Math., Vol.53,(1974), pp.249-315.

[21] C.N. Yang and R.L. Mills, Conservation of isotopic spin and isotopic gauge invariance, Phys. Rev., Vol.96, (1954),pp.191-195.

[22] M.F. Atiyah andR.S. Ward, Instants and algebraic geometry, Commun. Math. Phys., Vol.55, (1977),pp.117-124.

[23] A.H. Khater, D.K. Callebaut and S.M. Sayed, Exact solutions for some nonlinear evolution equations which describe pseudo-spherical surfaces, J. of Comput. and Appl. Math., Vol.189,(2006), pp.387-411.

[24] M.J. Ablowitz,S. Chakravarty and R. Halburd, The generlized Chazy equation and Schwarzian triangle functions, Asian J. Math., Vol.2,(1998), pp.619-624.

[25] M.J. Ablowitz,S. Chakravarty and R. Halburd, The generalized Chazy equation from the self-duality equations, Stud. Appl. Math., Vol.103,(1999), pp. $75-88$.

[26] M.J. Ablowitz,S. Chakravarty and R. Halburd, On Painleve and DarbouxHalphen type equations, in the Painleve property, One Century Later, edited By R. Conte, CRM Series in Mathematical Physics (Springer, Berlin), (1998).

[27] M.J. Ablowitz,S. Chakravarty and R. Halburd, Integrable systems and reductions of the self-dual Yang-Mills equations, J. Math. Phys., Vol.44,(2003), pp.3147-3173.

[28] M.J. Ablowitz,S. Chakravarty and L. A. Takhtajan, A self-dual Yang-Mills hierarchy and its reductions to integrable systems in $1+1$ and $2+1$ dimensions, Commun. Math. Phys., Vol.158,(1993), pp.289-314.

[29] S.M. Sayed and G.M. Gharib, Canonical reduction of Self-Dual Yang-Mills equations to Fitzhugh-Nagumo equation and exact solutions, Chaos, Solitons Fractals, Vol.39,(2009), pp.492-498.

[30] K. Konno and M. Wadati, Simple derivation of Bäcklund transformation from Riccati Form of inverse method, Progr. Theor. Phys., Vol.53,(1975), pp.1652-1656. 
[31] R. Sasaki, Soliton equations and pseudo-spherical surfaces, Nucl. Phys., Vol.B154,(1979), pp.343-35\%. 